\title{
Efficacy of intravesical chondroitin sulphate in treatment of interstitial cystitis/bladder pain syndrome (IC/BPS): Individual patient data (IPD) meta-analytical approach
}

\author{
Ammarin Thakkinstian, PhD;* J. Curtis Nickel, MD, FRCSC ${ }^{\dagger}$
}

*Section for Clinical Epidemiology and Biostatistics, Faculty of Medicine, Ramathibodi Hospital, Mahidol University, Bangkok, Thailand; †Department of Urology, Queens University, Kingston, ON

Cite as: Can Urol Assoc J 2013;7(5-6):195-200. http://dx.doi.org/10.5489/cuaj.1257

\section{Abstract}

Background: Raw data from 3 similar clinical trials were analyzed in this individual participant data (IPD) meta-analysis to define any possible efficacy of intravesical $2 \%$ chondroitin sulphate in IC/BPS. Methods: We pooled IPD from an open label and 2 small randomized placebo controlled trials assessing chondroitin sulphate in IC/BPS (similar inclusion/exclusion criteria, treatment, outcome assessment). Our primary objective was to compare rates of global response assessment (GRA) responsiveness between chondroitin sulphate and vehicle control. Secondary objectives compared the Interstitial Cystitis Symptom/Problem Index (ICSI/PI) total score and improvement rates, and average daily urine frequency. The treatment response was calculated for individual trials and pooled data using IPD meta-analysis for pooling proportions.

Results: In total, 213 patients were included in the pooling analysis. At the end of the treatment period, the overall GRA response rates were 43.2 (95\% Cl: $35.0,51.5)$ and $27.4(95 \% \mathrm{Cl}: 17.6,37.2)$ for the chondroitin sulphate and vehicle control groups, respectively. Pooled RR was 1.55 ( $p=0.014,95 \% \mathrm{Cl}: 1.09,2.22)$. The chance of being an ICSI responder was similarly $54 \%$ higher in the chondroitin sulphate group. The small decrease in total ICSI score and urine frequency between the two groups was less impressive (-0.8 and -0.5 points respectively) and not statistically significant.

Conclusions: Benefits from intravesical chondroitin sulphate treatment in IC/BPS patients can be confirmed by increasing the power of the available data using an IPD meta-analytical approach. However, disconnect between response rates and severity scores underline the importance of choosing the right patient for this organ-specific treatment.

\section{Introduction}

Interstitial cystitis/bladder pain syndrome (IC/BPS) is a noninfectious chronic bladder condition characterized by pelvic pain perceived to be related to the bladder and associated urinary storage symptoms (frequency, urgency, and nocturia).1,2 An important component of the urothelial mucin glycosaminoglycan (GAG) layer, ${ }^{3}$ which is believed to be abnormal in IC/BPS, is chondroitin sulfate. ${ }^{4} \mathrm{~A} 2.0 \%$ solution of sodium chondroitin sulfate in phosphate buffered saline (chondroitin sulphate, Tribute Pharmaceuticals [formerly Stellar Pharmaceuticals], Milton, ON) has been approved in Canada and Europe for intravesical instillation as a medical device for the replenishment of the GAG layer to relieve the symptoms of IC/BPS.

A number of uncontrolled, open-label clinical studies, previously conducted with intravesical chondroitin sulfate, ${ }^{5-7}$ reported large reductions in IC/BPS symptoms with no significant treatment safety issues. A prospective real-life clinical practice study (referred to in this report as "Open Label Study" or OLS) ${ }^{8}$ completed in 53 subjects, described a treatment responder rate of $47 \%$ after 6 once-weekly instillations and $60 \%$ were after an additional 4 monthly instillations. Two better designed randomized placebo controlled studies ${ }^{9,10}$ reported that patients treated with intravesical chondroitin sulfate had a greater chance of experiencing amelioration of their symptoms, however in both studies the treatment effect was not statistically significant. The first proof of concept study (referred to in this report as "RCT $\# 1$ "), ${ }^{9}$ a small trial, which enrolled 62 patients in total, reported a possible treatment effect with $39 \%$ of patients treated with 6 weekly chondroitin sulfate treatments showing clinical response compared to $23 \%$ of patients in the control group. A second controlled clinical trial (referred to as "RCT $\left.\# 2^{\prime \prime}\right)^{10}$ was undertaken to gain additional safety and effectiveness information in 98 IC/BPS subjects with intravesical $2 \%$ chondroitin sulfate using 8 weekly instillations over a 7-week period with post-treatment follow-up for an additional 19 weeks for a total study length of 26 weeks. Patients randomized to intravesical $2 \%$ chondroitin sulfate had a $43 \%$ response rate compared to a $34 \%$ response rate in the placebo group (difference was not statistically significant).

These 3 studies were designed (the 2 RCTs were purposely underpowered) to use the data to determine possible safety and efficacy information to decide on whether it would be prudent to continue investigating and using this intervention. 
While only 2 of the 3 clinical studies were RCTs and all used slightly different durations of therapy, they all enrolled similar patients, had the same treatment intervention and used the same assessment tools. This allowed us to combine the individual data sets which yielded more power and thus gave us a robust analysis using a meta-analytical approach. Such an analysis assisted us in defining any possible efficacy of intravesical $2 \%$ chondroitin sulphate in IC/BPS.

\section{Methods}

\section{Patients}

We analyzed raw data from 3 clinical trials assessing chondroitin sulphate in IC/BPS patients in this meta-analysis. ${ }^{8-10}$ The 3 clinical trials had similar inclusion/exclusion criteria, treatment (intravesical chondroitin sulphate) and outcome assessment questionnaires (global response assessment [GRA], O'Leary Sant Interstitial Cystitis Symptom [ICSI] and the Interstitial Cystitis Problem Index [ICPI] questionnaires and usual safety assessments). Voiding diaries to measure 24 -hour voiding frequency were included in RCT\#1 and $\# 2$, but voided volume was only collected for RCT\#2. None of the clinical trials included urodynamic assessment. One trial (OLS) was an open label study ${ }^{8}$ without control group, while the other 2 (RCT \#1 and \#2) $)^{9,10}$ were randomized placebo controlled trials. The duration of weekly intravesical chondroitin sulphate instillation were 6 weeks (plus 4 monthly follow-up instillations), 6 weeks and 8 weeks for OLS, RCT\# 1 and RCT \#2, respectively.

\section{Objectives}

The primary objective was to compare rates of GRA responsiveness between chondroitin sulphate and vehicle control in subjects with IC/BPS. The secondary objective was to compare rates of improvement of ICSI, ICSI total score, ICPI total score and improvement rates, ${ }^{11}$ and average daily urine frequency between chondroitin sulphate and vehicle control groups.

\section{Study endpoints}

The primary endpoint was treatment responsiveness, which was defined as moderate to marked improvement on the GRA scale at Week 10 for the OLS, Week 7 for the RCT \#1 and Week 11 for the RCT \#2. The GRA was employed as the primary endpoint in all 3 studies. The secondary endpoints were ICSI Improvement (responder), which was defined as a decrease by $30 \%$ of ICSI total scores from baseline, ICSI total score, ICPI improvement (responder), which was defined as a decrease by $30 \%$ of ICPI total scores from baseline, ICPI total score, and average daily urinary frequency according to a 2-day voiding diary These outcomes were measured at the end of treatment period and the end of study, which were Week 10 and Week 24 for OLS, Week 7 and Week 12 for RCT \#1, and Week 11 and Week 26 for RCT \#2. If data for the current visit were missing, the previous observed data were carried forward to replace missing data. This is known as the last observe carry forward (LOCF) technique.

\section{Statistical analysis}

For individual study, data were described using mean (or median where appropriate) and frequency (\%) for continuous and dichotomous data, respectively. Patients' characteristics at baseline were compared between treatment groups using t-test (or Mann-Whitney test) for continuous data and chi-square test for categorical data.

For dichotomous endpoints (i.e., the GRA response and ICSI/ICPI improvement/responder), a response rate for each study was estimated according to treatment groups and time at measurement. The rates were then pooled using metaanalysis for pooling proportions. Treatment effects, measured by risk ratio (RR) along with $95 \%$ confidence interval $(\mathrm{Cl})$, were then estimated. A binary regression model with adjusting cluster (study) effect was applied to assess overall treatment effects. The pooled RRs and 95\% Cls were estimated by exponential coefficients of treatments.

For continuous outcomes (i.e., ICSI, ICPI, average urine frequency), the mean difference between the 2 treatment groups was estimated for each study. Linear regression adjusted by study effect (cluster) was applied to assess treatment effects on continuous outcomes.

Modified intention to treat (ITT) and ITT were applied for RCT \#1 and RCT \#2 studies, respectively. All analyses were performed using STATA version 11.1. The $p$ values with twosided tests $<0.05$ were considered statistically significant.

\section{Results}

In total, 213 patients (53, 62 and 98 for OLS, RCT \#1 and RCT \#2, respectively) were included in this pooling analysis. Patients' characteristics at baseline, between treatment groups, for each study were similar (Table 1 ).

\section{GRA response rate}

We tallied the GRA response rate for each study (Table 2). At the end of the treatment period (Week 10 for OLS, Week 7 for RCT \#1, Week 11 for RCT \#2), the overall rates were $43.2(95 \% \mathrm{Cl}: 35.0,51.5)$ and $27.4(95 \% \mathrm{Cl}: 17.6,37.2)$ for the chondroitin sulphate and vehicle control groups, respectively. The pooled RR was 1.55 ( $p=0.014,95 \% \mathrm{Cl}$ : 1.09, 2.22 ) (i.e., the chance of having response to treatment was 


\begin{tabular}{|c|c|c|c|c|c|c|}
\hline \multirow{2}{*}{ Study } & \multirow{2}{*}{ Parameter } & \multicolumn{2}{|c|}{ Chondroitin sulphate } & \multicolumn{2}{|c|}{ Control } & \multirow{2}{*}{$p$ value } \\
\hline & & Mean & SD & Mean & SD & \\
\hline \multirow[t]{4}{*}{ RCT 1} & Age & 45.5 & 16.1 & 44.9 & 15.1 & 0.880 \\
\hline & ICSI & 13.8 & 3.6 & 14.8 & 3.0 & 0.258 \\
\hline & ICPI & 12.4 & 3.3 & 12.9 & 2.3 & 0.441 \\
\hline & Diary urine frequency & 16.4 & 4.8 & 17.6 & 6.3 & 0.398 \\
\hline \multirow[t]{4}{*}{ RCT 2} & Age & 45 & 14.7 & 46.6 & 13.9 & 0.585 \\
\hline & ICSI & 12.9 & 3.4 & 12.8 & 3.9 & 0.877 \\
\hline & ICPI & 12.4 & 2.7 & 11.7 & 2.9 & 0.245 \\
\hline & Diary urine frequency & 13.9 & 7.2 & 15.1 & 7.2 & 0.450 \\
\hline \multirow[t]{3}{*}{ Open-label* } & Age & 44 & 15.3 & 45.7 & 14.3 & 0.557 \\
\hline & ICSI & 14.0 & 3.5 & 13.6 & 3.4 & 0.513 \\
\hline & ICPI & 12.6 & 2.6 & 12.2 & 2.8 & 0.408 \\
\hline
\end{tabular}

*Controls were combined RCT 1 and RCT 2 controls. RCT: randomized control trial; ICSI: Interstitial Cystitis Symptom Index; ICPI: Interstitial Cystitis Problem Index; SD: standard deviation.

$55 \%$ significantly higher in the chondroitin sulphate group than in the vehicle control group).

The pooled response rates at the end of the study (i.e., Week 24, 12 and 26 for OLS, RCT \#1, and RCT \#2 respectively) were $43.3(95 \% \mathrm{Cl}: 17.3,69.2)$ and $24.6(95 \% \mathrm{Cl}$ : $14.7,34.5)$ for chondroitin sulphate and vehicle control groups, respectively. The chance of having responsiveness was found to be $80 \%(\mathrm{RR}=1.80, p=0.070,95 \% \mathrm{Cl}$ : 0.95, 3.40) higher, but not significant in the chondroitin sulphate group compared with the vehicle control group.

ICSI

\section{ICSI improvement}

ICSI improvements at the end of the treatment period were significantly higher in the chondroitin sulphate than the vehicle control group with the rates of $48.0 \%(95 \% \mathrm{Cl}$ : $34.9,61.0)$ and $30.3 \%(95 \% \mathrm{Cl}: 15.7,44.9)$, respectively (Table 3). Therefore, the chance of having disease-specific improvement was $54 \%(\mathrm{RR}=1.54, p=0.033,95 \% \mathrm{Cl}: 1.04$, $2.30)$ significantly higher in the chondroitin sulphate than in the vehicle control group. The improvement was consistent until the end of the study period with the pooled RR of 1.34 $(p=0.028,95 \% \mathrm{Cl}: 1.03,1.73)$.

\section{ICSI total score}

The mean ICSI total scores have been described according to treatments and time at assessment (Table 4). A mean difference was estimated for each study and then pooled. The overall mean difference at the end of treatment period was -0.8 ( $p=0.404,95 \% \mathrm{Cl}:-4.0,2.4)$, In other words, the ICSI is 0.8 units lower, but not significant in the chondroitin sulphate than in the vehicle control group. The overall mean difference at the end of this study was 0.5 ( $p=0.743,95 \%$ $\mathrm{Cl}$ : $-5.8,4.8)$ higher, but not significant in the chondroitin sulphate compared with the vehicle control group.

\section{ICPI}

\section{ICPI improvement}

The overall ICPI responder rates at the end of treatments were 50.7 (95\% Cl: 42.3, 59.1) and 41.8 (95\% Cl: 30.9, 52.6) for the chondroitin sulphate and the vehicle control groups, respectively (Table 5 ). The pooled RR was 1.21 $(p=0.006,95 \% \mathrm{Cl}: 1.06,1.40)$. In other words, the chance of having ICPI improvement was $21 \%$ significantly higher in the chondroitin sulphate than in the vehicle control group. The long-term effects at the end of study period decreased with the pooled RR of 1.15 ( $p=0.565,95 \% \mathrm{Cl}: 0.72,1.83)$.

Table 2. Comparison of GRA response rates between chondroitin sulphate and control groups

\begin{tabular}{|c|c|c|c|c|c|c|c|c|c|c|c|c|c|c|}
\hline \multirow{3}{*}{ Study } & \multicolumn{7}{|c|}{ End of treatment } & \multicolumn{7}{|c|}{ End of study } \\
\hline & \multicolumn{3}{|c|}{ Chondroitin sulphate } & \multicolumn{3}{|c|}{ Control } & \multirow{2}{*}{$\begin{array}{c}\text { RR } \\
\text { (95\% Cl) } \\
\end{array}$} & \multicolumn{3}{|c|}{ Chondroitin sulphate } & \multicolumn{3}{|c|}{ Control } & \multirow{2}{*}{$\begin{array}{c}\text { RR } \\
(95 \% \mathrm{Cl}) \\
\end{array}$} \\
\hline & $\mathbf{n}$ & $\mathbf{R}$ & Rate & $\mathbf{n}$ & $\mathbf{R}$ & Rate & & $\mathbf{n}$ & $\mathbf{R}$ & Rate & $\mathbf{n}$ & $\mathbf{R}$ & Rate (\%) & \\
\hline RCT 1 & 33 & 13 & $39.4 \%$ & 31 & 7 & $22.6 \%$ & $1.74(0.80,3.79)$ & 30 & 13 & $43.3 \%$ & 30 & 7 & 23.3 & $1.85(0.86,3.99)$ \\
\hline RCT 2 & 50 & 19 & $38 \%$ & 48 & 15 & $31.3 \%$ & $1.22(0.70,2.11)$ & 44 & 10 & $22.7 \%$ & 43 & 11 & 25.6 & $0.89(0.42,1.87)$ \\
\hline OLS & 53 & 27 & $50.9 \%$ & - & - & - & & 50 & 32 & $64.0 \%$ & - & - & - & \\
\hline Pooled & & & $43.2 \%$ & & & $27.4 \%$ & 1.55 & & & $43.3 \%$ & & & 24.6 & 1.80 \\
\hline $95 \% \mathrm{Cl}$ & & & $35.0,51.5$ & & & $17.6,37.2$ & $1.09,2.22 *$ & & & $17.3,69.2$ & & & $14.7,34.5$ & $0.95,3.40 * *$ \\
\hline
\end{tabular}

${ }^{*} p=0.014 ;{ }^{* *} p=0.070$; GRA: Global response assessment; R: response; Cl: confidence interval; RR: risk ratio; RCT: randomized control trial; OLS: open-label study. 
Table 3. Comparison of ICSI responder rates between chondroitin sulphate and control groups at end of treatment period and end of study

\begin{tabular}{|c|c|c|c|c|c|c|c|c|c|c|c|c|c|c|}
\hline \multirow{3}{*}{ Study } & \multicolumn{7}{|c|}{ End of treatment } & \multicolumn{7}{|c|}{ End of study } \\
\hline & \multicolumn{3}{|c|}{ Chondroitin sulphate } & \multicolumn{3}{|c|}{ Control } & \multirow{2}{*}{$\begin{array}{c}\text { RR } \\
(95 \% \mathrm{Cl})\end{array}$} & \multicolumn{3}{|c|}{ Chondroitin sulphate } & \multicolumn{3}{|c|}{ Control } & \multirow{2}{*}{$\begin{array}{c}\text { RR } \\
(95 \% \mathrm{Cl})\end{array}$} \\
\hline & $\mathbf{n}$ & $\mathbf{R}$ & Rate & $\mathbf{n}$ & $\mathbf{R}$ & Rate & & $\mathbf{n}$ & $\mathbf{R}$ & Rate & $\mathbf{n}$ & $\mathbf{R}$ & Rate (\%) & \\
\hline RCT 1 & 33 & 12 & $36.4 \%$ & 31 & 7 & $22.6 \%$ & $\begin{array}{c}1.66 \\
(0.73,3.55)\end{array}$ & 29 & 12 & $41.4 \%$ & 30 & 10 & $33.3 \%$ & $\begin{array}{c}1.24 \\
(0.63,2.42)\end{array}$ \\
\hline RCT 2 & 50 & 23 & $46 \%$ & 48 & 18 & $37.5 \%$ & $\begin{array}{c}1.23 \\
(0.76,1.97)\end{array}$ & 44 & 19 & $43.2 \%$ & 44 & 17 & $38.6 \%$ & $\begin{array}{c}1.12 \\
(0.68,1.85)\end{array}$ \\
\hline OLS & 53 & 31 & $59.6 \%$ & - & - & - & - & 50 & 29 & $58.0 \%$ & - & - & - & - \\
\hline $\begin{array}{l}\text { Pooled } \\
\text { (95\% Cl) }\end{array}$ & & & $\begin{array}{c}48.0 \% \\
(34.9,61.0)\end{array}$ & & & $\begin{array}{c}30.3 \% \\
(15.7,44.9) \\
\end{array}$ & $\begin{array}{c}1.54 \\
(1.04,2.30)^{*}\end{array}$ & & & $\begin{array}{c}48.4 \% \\
(37.7,59.1) \\
\end{array}$ & & & $\begin{array}{c}36.4 \% \\
(34.3,41.5) \\
\end{array}$ & $\begin{array}{c}1.34 \\
(1.03,1.73)^{* *}\end{array}$ \\
\hline
\end{tabular}

\section{ICPI total score}

The mean differences of ICPI total scores were estimated (table 6). The pooled mean difference was $-0.5(p=0.256$, $95 \% \mathrm{Cl}:(-1.7,0.8)$, i.e., the ICPI total score was 0.5 unit lower but not significant in the chondroitin sulphate compared with the vehicle group. The pooled mean difference at the end study period was -0.3 ( $p=0.795,95 \% \mathrm{Cl}:-5.2,4.5$ ).

\section{Average daily urine frequency}

The mean difference of average daily urine frequency was estimated for each study (Table 7). The pooled mean difference was $-0.5(p=0.613,95 \% \mathrm{Cl}:-8.8,7.9)$. The average daily-urine frequency was 0.5 times lower in the chondroitin sulphate than the vehicle control group. In RCT \#2 (voided volume was collected in voiding diaries), the average urine per void decreased slightly in the control group (-0.2 cc) and increased moderately in the chondroitin sulphate group (+15.0 cc).

\section{Safety}

Overall, the treatment was very safe. In the OLS, 53\% of subjects reported an adverse event, while adverse events were reported in $76.9 \%$ and $70.4 \%$ of the subjects enrolled in RCT \#1 and RCT \#2, respectively (with no difference between control and active treatment groups). All serious adverse events were unrelated to treatment. Possible treatment related adverse events were reported in 30.8\%, 16.9\% and $7.6 \%$ in OLS, RCT $\# 1$ and RCT $\# 2$, respectively. These adverse events, which were reported as mild or moderate, occurred more frequently in the control group compared to the active treatment group in the two RCTs. Urinary tract infections (defined as new onset symptoms associated with significant bacteriuria) were reported in 6 patients in the OLS and 3 patients in RCT \#1 (all in control group).

\section{Discussion}

Meta-analysis is a statistical tool that can be used to obtain a quantitative estimate of the effect of a particular intervention from the effects reported in many studies. Since there are 3 small inconclusive studies evaluating chondroitin sulphate, it would seem that an individual patient data (IPD) metaanalytical approach would be the best way to determine if there is a significant efficacy signal in the pooled data. The main purpose of this IPD meta-analysis was to increase statistical power for primary outcomes from the data from the 3 available clinical trials in a cost-effective manner to determine whether larger and long-term studies should be contemplated.

The 3 studies to date (OLS, RCT \#1 and RCT \#2) all indicated that chondroitin sulphate may benefit patients with IC/BPS. ${ }^{8-10}$ This IPD meta-analysis confirms that chon-

Table 4. Comparison of mean ICSI total scores between chondroitin sulphate and control groups at end of treatment period and end of study

\begin{tabular}{|c|c|c|c|c|c|c|c|c|c|c|}
\hline \multirow{3}{*}{ Study } & \multicolumn{5}{|c|}{ End of treatment } & \multicolumn{5}{|c|}{ End of study } \\
\hline & \multicolumn{2}{|c|}{ Condroitin sulphate } & \multicolumn{2}{|c|}{ Control } & \multirow{2}{*}{$\begin{array}{c}\text { Difference } \\
(95 \% \mathrm{Cl}) \\
\end{array}$} & \multicolumn{2}{|c|}{ Condroitin sulphate } & \multicolumn{2}{|c|}{ Control } & \multirow{2}{*}{$\begin{array}{c}\text { Difference } \\
(95 \% \mathrm{Cl}) \\
\end{array}$} \\
\hline & $\mathbf{n}$ & $\mathbf{R}$ & $\mathbf{n}$ & $\mathbf{R}$ & & $\mathbf{n}$ & $\mathbf{R}$ & $\mathbf{n}$ & $\mathbf{R}$ & \\
\hline \multicolumn{11}{|c|}{ ICSI after treatments } \\
\hline RCT 1 & 33 & $11.0(4.8)$ & 31 & $11.9(3.8)$ & $-0.9(-3.1,1.2)$ & 29 & $11.1(5.0)$ & 30 & $11.5(4.5)$ & $-0.4(-2.9,2.06)$ \\
\hline RCT 2 & 50 & $9.7(5.0)$ & 48 & $9.7(4.9)$ & $0.0(-2.0,2.0)$ & 44 & $10.6(5.1)$ & 44 & $9.4(4.3)$ & $1.2(-0.8,3.1)$ \\
\hline OLS & 53 & $9.2(4.2)$ & - & - & - & 53 & $8.3(5.0)$ & - & - & - \\
\hline Pooled (95\% Cl) & & & & & $-0.8(-4.0,2.4)^{*}$ & & & & & $0.5(-5.8,4.8)^{* *}$ \\
\hline
\end{tabular}

${ }^{*} p=0.404 ;{ }^{* *} p=0.743$. ICSI: Interstitial Cystitis Symptom Index; Cl: confidence interval; RCT: randomized control trial; OLS: open-label study. 
Table 5. Comparison of ICPI responder rates between chondroitin sulphate and control groups at end of treatment period and end of study

\begin{tabular}{|c|c|c|c|c|c|c|c|c|c|c|c|c|c|c|}
\hline \multirow{3}{*}{ Study } & \multicolumn{7}{|c|}{ End of treatment } & \multicolumn{7}{|c|}{ End of study } \\
\hline & \multicolumn{3}{|c|}{ Chondroitin sulphate } & \multicolumn{3}{|c|}{ Control } & \multirow{2}{*}{$\begin{array}{c}\text { RR } \\
(95 \% \mathrm{CI}) \\
\end{array}$} & \multicolumn{3}{|c|}{ Chondroitin sulphate } & \multicolumn{3}{|c|}{ Control } & \multirow{2}{*}{$\begin{array}{c}\text { RR } \\
(95 \% \mathrm{Cl})\end{array}$} \\
\hline & $\mathbf{n}$ & $\mathbf{R}$ & Rate & $\mathbf{n}$ & $\mathbf{R}$ & Rate & & $\mathbf{n}$ & $\mathbf{R}$ & Rate & $\mathbf{n}$ & $\mathbf{R}$ & Rate (\%) & \\
\hline RCT 1 & 33 & 14 & $42.4 \%$ & 31 & 13 & $41.9 \%$ & $\begin{array}{c}1.01 \\
(0.57,1.80)\end{array}$ & 29 & 11 & $37.9 \%$ & 30 & 9 & 30.0 & $\begin{array}{c}1.26 \\
(0.62,2.59)\end{array}$ \\
\hline RCT 2 & 50 & 28 & $56.0 \%$ & 48 & 20 & $41.7 \%$ & $\begin{array}{c}1.34 \\
(0.89,2.04)\end{array}$ & 44 & 18 & $40.9 \%$ & 43 & 21 & 48.8 & $\begin{array}{c}0.84 \\
(0.52,1.33)\end{array}$ \\
\hline OLS & 51 & 26 & $50.0 \%$ & - & - & - & & 50 & 29 & $58.0 \%$ & - & - & - & \\
\hline $\begin{array}{l}\text { Pooled } \\
(95 \% \mathrm{Cl})\end{array}$ & & & $\begin{array}{c}50.7 \% \\
(42.3,59.1)\end{array}$ & & & $\begin{array}{c}41.8 \% \\
(30.9,52.6)\end{array}$ & $\begin{array}{c}1.21 \\
(1.06,1.40)^{*}\end{array}$ & & & $\begin{array}{c}46.3 \% \\
(33.7,59.0)\end{array}$ & & & $\begin{array}{c}39.7 \\
(21.3,58.2)\end{array}$ & $\begin{array}{c}1.15 \\
(0.72,1.83)^{* *}\end{array}$ \\
\hline
\end{tabular}

droitin sulphate does indeed provide significantly more benefit than placebo. At the end of the treatment period, patients who had experienced chondroitin sulphate therapy had a response rate (rated their improvement as moderate or marked) of $43 \%$ compared to $27 \%$ for placebo treated patients. This meant that the chance of having becoming a responder with chondroitin sulphate was $55 \%$ significantly higher than with placebo treatment. While the chance of being a responder at the end of the study period (different duration for 3 studies) was $80 \%$ higher in the chondroitin sulphate groups compared to the placebo group, this was not statistically significant (certainly arguably clinically significant). This global response was confirmed using an ICSI responder definition. In this case, the chance of being a responder was $54 \%$ higher in the chondroitin sulphate group (48\% compared to $30 \%$ in the chondroitin sulphate and placebo groups, respectively). The small beneficial difference in decrease in total mean ICSI score between the 2 groups was present, but much less impressive (-0.8 points) and not statistically significant. Similar findings were seen for ICPI; although there was significant benefit with the responder analysis, the difference in total score was not statistically significant. There was a decrease in mean average daily urine frequency in the chondroitin sulphate group compared to the placebo group, but it was very modest (-0.5) and not statistically significant.

A number of limitations of this analysis are evident. One of the studies was not a randomized placebo controlled study, and while the patient populations are similar between studies, response bias is a potential factor. The 3 clinical trials employed similar weekly treatment regimens with the same active treatment; however, the treatment durations for primary outcome and end of study were different. They were similar enough to allow us to combine primary outcome and longer term end of study data in our analysis. This IPD meta-analysis met the minimum standards required, including prospective protocols, comparable definitions of key outcomes, quality control of data, and inclusion of all patients from all trials in the final analysis.

This analysis confirms that chondroitin sulphate is an effective therapy for some patients with IC/BPS. While the chance of being a responder was clearly statistically and clinically significantly better for chondroitin sulphate patient compared to placebo, the disconnect between the 3 responder analyses (very clear difference between chondroitin sulphate and controls) and the decrease in total disease specific scores (modest and not significant) indicate that we must do a better job of determining who will do well with this GAG replacement therapy. A similar disconnect between response rates and total score impact was observed in a recent major meta-analysis examining multiple treatment options for men with chronic prostatitis/chronic pelvic pain syndrome. ${ }^{12}$ The modest improvement seen using a monotherapy approach in IC/BPS was recently confirmed in a large meta-analysis of treatment outcomes in IC/BPS. ${ }^{13}$ If we were able to better select more appropriate patients for this therapy, we would see not only an increased chance of the patient being classified as a chondroitin sulphate

Table 6. Comparison of mean ICPI total scores between chondroitin sulphate and control groups at end of treatment period and end of study

\begin{tabular}{|c|c|c|c|c|c|c|c|c|c|c|}
\hline \multirow{3}{*}{ Study } & \multicolumn{5}{|c|}{ End of treatment } & \multicolumn{5}{|c|}{ End of study } \\
\hline & \multicolumn{2}{|c|}{ Condroitin sulphate } & \multicolumn{2}{|c|}{ Control } & \multirow[t]{2}{*}{ Difference } & \multicolumn{2}{|c|}{ Condroitin sulphate } & \multicolumn{2}{|c|}{ Control } & \multirow[t]{2}{*}{ Difference } \\
\hline & $\mathbf{N}$ & Mean (SD) & $\mathbf{N}$ & Mean (SD) & & $\mathbf{N}$ & Mean (SD) & $\mathbf{N}$ & Mean (SD) & \\
\hline RCT 1 & 33 & $9.5(4.5)$ & 32 & $9.9(4.4)$ & $-0.4(-2.6,1.8)$ & 29 & $9.3(4.8)$ & 30 & $9.9(4.2)$ & $-0.6(-2.9,1.8)$ \\
\hline RCT 2 & 50 & $7.9(4.6)$ & 48 & $8.3(4.5)$ & $-0.4(-2.2,1.5)$ & 44 & $9.1(4.8)$ & 43 & $7.9(4.2)$ & $1.2(-0.7,3.1)$ \\
\hline OLS & 51 & $8.4(3.9)$ & - & - & - & 50 & $7.2(4.4)$ & - & - & - \\
\hline Pooled & & & & & $-0.5(-1.7,0.8)^{*}$ & & & & & $-0.3(-5.2,4.5)^{* *}$ \\
\hline
\end{tabular}

${ }^{*} p=0.256 ;{ }^{* *} p=0.795 ;$ ICSI: Interstitial Cystitis Symptom Index; ICPI: Interstitial Cystitis Problem Index; SD: standard deviation; RCT: randomized control trial; OLS: open-label study. 


\begin{tabular}{|c|c|c|c|c|c|}
\hline \multirow{3}{*}{ Study } & \multicolumn{5}{|c|}{ End of treatment } \\
\hline & \multicolumn{2}{|c|}{ Chondroitin sulphate } & \multicolumn{2}{|r|}{ Control } & \multirow{2}{*}{$\begin{array}{c}\text { Difference } \\
(95 \% \mathrm{Cl}) \\
\end{array}$} \\
\hline & $\mathbf{N}$ & Mean (SD) & $\mathbf{N}$ & Mean (SD) & \\
\hline RCT 1 & 33 & $14.2(4.2)$ & 32 & $15.4(7.6)$ & $-1.2(-4.4,1.8)$ \\
\hline RCT 2 & 50 & $12.4(7.6)$ & 48 & $12.3(6.5)$ & $0.1(-4.4,1.8)$ \\
\hline Pooled & & & & & $-0.5(-8.8,7.9)^{*}$ \\
\hline
\end{tabular}

responder, but also we would expect to see a better overall disease response in terms of decrease of IC/BPS scores in the selected populations. ${ }^{14}$ Examples of positive selection criteria likely will be those patients with organ-specific features of IC/BPS, such as classic cystoscopic findings (glomerulations, Hunner's lesions), perhaps biopsies (showing inflammation) and/or provocative testing, such as the potassium sensitivity test (which is an indirect test for bladder permeability). It is hoped that better biomarkers (likely urine but perhaps serum/blood) that are being evaluated by the NIH/NIDDK in the MAPP study ${ }^{15}$ will allow for better patient differentiation and improved therapeutic targeting.

\section{Conclusion}

It appears that the benefits obtained with chondroitin sulphate in IC/BPS patients, suggested but not statistically proven in small underpowered clinical trials, can be confirmed by increasing the power of the available data using a metaanalytical approach.

Competing interests: Dr. Nickel was an investigator and consultant for Stellar Pharmaceuticals and Watson Pharmaceuticals. Dr. Thakkinstian was a consultant for Stellar Pharmaceuticals. These companies had no input into the design, writing or publication of this analysis.

\section{References}

1. Carr LK, Corcos J, Nickel JC, et al. Diagnosis of interstitial cystitis. Can Urol Assoc J 2009;3:81-6.

2. Hanno PM, Allen D, Burks JQ, et al. AUA Guidelines for the diagnosis and treatment of intersititial cystitis/ bladder pain syndrome. J Urol 2111:185:2162-70.

3. Parsons CL. Bladder Surface Glycosaminoglycans. Efficient Mechanism of Environmental Adaptation. Urol 1986;27:9-14

4. Hurst RE, Roy J, Min RK, et al. A deficit of chondroitin sulfate proteoglycans of the bladder uroepithelium in interstitial cystitis. Urol 1996:48:817-21. http://dx.doi.org/10.1016/S0090-4295(96)00322-6

5. Sorensen RB. Chondroitin sulphate in the treatment of intersititial cystitis and chronic inflammatory disease of the urinary bladder. Eur Urol 2003(Suppl 2):16-8.

6. Steinhoff $G$ : The efficacy of chondroitin sulphate in treating interstitial cystitis. Europ Urol Suppl 2003;2:146. http://dx.doi.org/10.1016/S1569-9056(03)00035-6

7. Nordling J, van Ophoven A. Intravesical Glysosaminoglycan replenishment with chondroitin sulfate in chronic forms of cystitis. Drug Research 2008;58:328-35.

8. Nickel JC, Egerdie B, Downey J, et al. A Real-Life Multi-Centre Clinical Practice Study to Evaluate the Efficacy and Safety of Intravesical Chondroitin Sulfate for the Treatment of Intersititial Cystitis. BJU Int 2008;103:56-60. http://dx.doi.org/10.1111/j.1464-410X.2008.08028.x

9. Nickel JC, Egerdie RB, Steinhoff G, et al. A Multi-Center, Randomized, Double-Blind, Parallel Group Pilot Evaluation of the Efficacy and Safety of Intravesical Sodium Chondroitin Sulfate versus Vehicle Control in Patients with Intersitital Cystitis/Painful Bladder Syndrome. Urol 2010;76:804-9.

10. Nickel JC, Hanno P, Kumar K, et al. A Second Multi-Center, Randomized, Double-Blind, Parallel Group Evaluation of the Effectiveness and Safety of Intravesical Sodium Chondroitin Sulfate Compared to Inactive Vehicle Control in Subjects with Interstitial Cystitis/Bladder Pain Syndrome. Urology 2012; in press.

11. O'Leary MP, Sant GR, Fowler FJ, et al. The Intersititial Cystitis Symptom Index and Problem Index. Urol 1997;49:58-63. http://dx.doi.org/10.1016/S0090-4295(99)80333-1

12. Anothaisintawee T, Attia J, Nickel JC, et al. The Management of Chronic Prostatitis/Chronic Pelvic Pain Syndrome: A systematic review and network meta-analysis. JAMA 2011;305:78-86. http://dx.doi. org/10.1001/jama.2010.1913

13. Giannantoni A, Bini V, Dmochowski R, et al. Contemporary Management of the Painful Bladder. Eur Urol 2012;61:29-53. http://dx.doi.org/10.1016/i.eururo.2011.07.069

14. Nickel JC, Shoskes D, Irvine-Bird K. Clinical Phenotyping of Women with Interstitial Cystitis/Painful Bladder Syndrome (IC/PBS): A Key to Classification and Potentially Improved Management. J Urol 2009;82:15560. http://dx.doi.org/10.1016/i.juro.2009.02.122

15. Multi-Disciplinary Approach to the Study of Chronic Pelvic Pain. http://www.mappnetwork.org. Accessed May 14, 2013.

Correspondence: Dr. J. Curtis Nickel, Department of Urology, Queens University, Kingston, ON K7L 2V7; icn@queensu.ca

This paper has been peer-reviewed. 